\title{
TESTÍCULO INTRAABDOMINAL Y SU MANEJO LAPAROSCÓPICO: 4 AÑOS DE EXPERIENCIA EN UN CENTRO ÚNICO
} INTRA-ABDOMINAL TESTICLEAND ITS LAPAROSCOPIC MANAGEMENT: 4 YEARS OF EXPERIENCE IN A SINGLE CENTER

\author{
TESTÍCULO INTRA-ABDOMINALE SEU MANEJO LAPAROSCÓPICO: \\ 4 ANOS DE EXPERIÊNCIA EM UMÚNICO CENTRO
}

\author{
JORGE OCAÑA GARCÍA', JULIORS GONZÁLES NAVARRO', VICENTE SALINAS SALINAS', JORGE OCAÑA DOMÍNGUEZ1 \\ ${ }^{1}$ Hospital de niños Roberto Gilbert Elizalde, Guayaquil, Ecuador
}

RESUMEN

Fundamento: el testículo no descendido se diagnostica desde el nacimiento o en las primeras consultas con el pediatra; se considera la malformación urogenital más frecuente en el varón. Cuando el testículo no puede palparse, debe consultarse a urología pediátrica y/o cirugía pediátrica, ya que esta condición puede acarrear las siguientes complicaciones: atrofia, torsión testicular, hernia inguinal sintomática o asintomática, y la posibilidad de que el defecto degenere hasta tejido neoplásico. Objetivo: evaluar el papel terapéutico de la laparoscopía en testículos no palpables en pacientes en el hospital de niños Dr. Roberto GilbertE. en la ciudad de Guayaquil, Ecuador. Métodos: Se realizó un estudio de prevalencia. Se identificaron 639 pacientes con testículos no descendidos y 37 de este grupo con testículo no palpable, 4 con afectación bilateral y 33 con testículos unilaterales impalpables que fueron manejados posteriormente por laparoscopía. Se documentaron detalles demográficos, lado del testículo impalpable, localización, hallazgos clínicos y laparoscópicos, hallazgos anatomopatológicos, intervención operativa y resultados. Resultados: la distribución del grupo etario en: $<1$ año, 1 a 5 años, 6 a 10 años y $>10$ años; en los cuales se vieron afectados $3(8 \%)$, 18(49\%), 11(30\%) y 5(14\%) pacientes respectivamente. En cuanto a la localización del testículo durante la valoración laparoscópica se encontraron $15(37 \%)$ a nivel del anillo inguinal interno y $26(63 \%)$ intrabdominales. La intervención operativa en base a los criterios previamente mencionados fueron 20(49\%) orquidopexia sin sección de los vasos espermáticos, 11(27\%) Fowler-Stephen y 10(24\%) orquiectomías. Durante el análisis de la anatomía patológica se encontraron 7(17\%) testículos evanescentes, 3(7\%)atróficos, $5(12 \%)$ hipotróficos, 22(54\%) hipoplásicos y 4(10\%)normales. Por último, 2(18\%) de los 11 pacientes operados con técnica de Fowler-Stephen presentaron atrofia. Conclusión: la laparoscopía es una herramienta extremadamente útil y modalidad segura tanto para el diagnóstico como para la gestión de testículos no palpables. Además de ser un excelente indicador intraoperatorio para decidir sobre el tipo de orquidopexia en base a la localización del testículo y las características morfológicas que presente el mismo. En todos los casos, es necesario un seguimiento a largo plazo para controlar el estado del testículo después de la cirugía.

PALABRAS CLAVE: criptorquidismo, laparoscopía, pediatría, intraabdominal, orquidopexia.

ABSTRACT

Background: the undescended testicle is diagnosed from birth or in first visits with the pediatrician; it is considered the most common urogenital malformation in men. When the testicle cannot be palpated, pediatric urology and/or pediatric surgery should take advice, this condition can lead to the following complications: atrophy, testicular torsion, symptomatic or asymptomatic inguinal hernia, and the possibility that the defect degenerates to neoplastic tissue. Objective: evaluate the therapeutic role of laparoscopy in non-palpable testicles in patients at the Dr. Roberto GilbertE. children's hospital in the city of Guayaquil, Ecuador. Methods: A prevalence study was carried out. We identified 639 patients with undescended testicles and 37 of this group with a non-palpable testis, 4 with bilateral involvement, and 33 with impalpable unilateral testicles that were subsequently managed by laparoscopy. Demographic details, impalpable testis side, location, clinical and laparoscopic findings, pathological findings, operative intervention, and results were documented. Results: the distribution of the age group in: $<1$ year, 1 to 5 years, 6 to 10 years and $>10$ years; in which $3(8 \%)$, 18 $(49 \%), 11(30 \%)$ and $5(14 \%)$ patients were affected respectively. Regarding the location of the testis during the laparoscopic evaluation, $15(37 \%)$ were found, at the level of the internal inguinal ring and $26(63 \%)$ intra-abdominal. The operative intervention based on the previously mentioned criteria were $20(49 \%)$ orchidopexy without a section of the spermatic vessels, 11 (27\%) Fowler-Stephen, and $10(24 \%)$ orchiectomies. During the analysis of the pathological anatomy, 7 (17\%) evanescent testes were found: 3 $(7 \%)$ atrophy, $5(12 \%)$ hypotrophy, 22 (54\%) hypoplastic, and $4(10 \%)$ normal. Finally, $2(18 \%)$ of the 11 patients operated on with the Fowler-Stephen technique presented atrophy. Conclusion: the laparoscopy is an extremely useful tool and a safe modality, both for the diagnosis and the management of non-palpable testicles. Besides, being an excellent intraoperative indicator to decide on the type of orchidopexy based on the location of the testicle and its morphological characteristics. In all cases, long-term follow-up is necessary to monitor the condition of the testicle after surgery.

KEYWORDS: cryptorchidism, laparoscopy, pediatrics, intra-abdominal, orchidopexy.

RESUMO

Antecedentes: o testículo não descido é diagnosticado desde o nascimento ou nas primeiras consultas com o pediatra; é considerada a malformação urogenital mais comum em homens. Quando o testículo não pode ser palpado, deve-se consultar a urologia pediátrica e / ou cirurgia pediátrica, pois essa condição pode levar às seguintes complicações: atrofia, torção testicular, hérnia inguinal sintomática ou assintomática e possibilidade de degeneração do defeito em tecido neoplásico. Objetivo: avaliar 0 papel terapêutico da laparoscopia em testículos não palpáveis em pacientes do hospital infantil Dr. Roberto GilbertE. na cidade de Guayaquil, Equador. Métodos: foi realizado um estudo de prevalência. Identificamos 639 pacientes com testículos não descidos e 37 desse grupo com testículos não palpáveis, 4 com envolvimento bilateral e 33 com testículos unilaterais impalpáveis que foram posteriormente tratados por laparoscopia. Detalhes demográficos, lado impalpável do testículo, localização, achados clínicose laparoscópicos, achados patológicos, intervenção cirúrgica e resultados foram documentados. Resultados: adistribuição da faixa etária em: $<1$ ano, 1 a 5 anos, 6 a 10 anose $>$ 10 anos; em que $3(8 \%), 18$ (49\%), 11 (30\%) e 5 (14\%) pacientes foram afetados, respectivamente. Em relaçãoà localização do testículo, durante a avaliação laparoscópica, $15(37 \%)$ foram encontrados ao nível do anel inguinal interno e 26 (63\%) intra-abdominal. A intervenção operatória com base nos critérios citados anteriormente foram 20 (49\%) orquiectomias sem secção dos vasos espermáticos, 11 (27\%) Fowler-Stephene 10 (24\%) orquiectomias. Durante a análise da anatomia patológica, foram encontrados $7(17 \%)$ testículos evanescentes, $3(7 \%)$ atróficos, $5(12 \%)$ hipotróficos, 22 (54\%) hipoplásicos e $4(10 \%)$ normais. Por fim, 2 (18\%) dos 11 pacientes operados pela técnica de Fowler-Stephen apresentaram atrofia. Conclusão: a laparoscopia é uma ferramenta extremamente útil e uma modalidade segura tanto para o diagnóstico quanto para o manejo de testícul os não palpáveis. Além de ser um excelente indicador intraoperatório para decidir o tipo de orquidopexia com base na localização do testículo e suas características morfológicas. Em todos os casos, énecessário um acompanhamento de longo prazo para monitorar a condição do testículo após a cirurgia. PALAVRAS-CHAVE: criptorquidia, laparoscopia, pediatria, intra-abdominal, orquidopexia. 
INTRODUCCIÓN

En aproximadamente 2a 5\% de los hombres, uno o ambos testículos no logran descender al escroto al momento del nacimiento. ${ }^{1,2}$ En muchos casos los testículos descenderán espontáneamente durante el primer año, pero aproximadamente un $23 \%$ permanecerá en criptorquidismo sin corrección quirúrgica. ${ }^{3}$ De estos, alrededor del $20 \%$ no son palpables y pueden estar localizados dentro del abdomen. ${ }^{4}$ Si no se corrige, el criptorquidismo y los testículos intraabdominales (TIAb) en particular, pueden conducir a un deterioro de la fertilidad. . $^{5-10}$ Con la corrección temprana, este riesgo se puede reducir o incluso ser eliminado. ${ }^{11,12}$

Sin embargo, la corrección quirúrgica del TIAb a menudo es técnicamente desafiante, ya que los vasos testiculares pueden no tener la longitud suficiente para permitir la movilización del testículo al escroto.

Existe una variedad de técnicas para la corrección quirúrgica de la criptorquidia. La más común es la orquidopexia primaria en la que el cirujano moviliza a los testículos no descendidos y los ubica en el escroto.

Sin embargo, en el caso de un TIAb, el cordón espermático corto puede complicar este procedimiento; así, en 1959, Fowler y Stephens propusieron una técnica alternativa, conocida como la orquidopexia de Fowler-Stephens (FSO), en la cual el cirujano divide el cordón espermático para colocar el testículo de manera apropiada. ${ }^{13}$

Ransley Modificó esta técnica en 1984, realizando la cirugía en dos etapas para mejorar supuestamente la viabilidad del testículo. ${ }^{14}$ Desde que Cortesi y cols en 1976 utilizaron la laparoscopía como técnica diagnóstica en los casos de testes no palpables, se ha demostrado que es la mejor exploración por encima de las pruebas de imagen. ${ }^{15,16}$

El objetivo principal de este trabajo es evaluar el papel terapéutico de la laparoscopia en testículos no descendidos no palpables en pacientes en el hospital de niños Dr. Roberto Gilbert Elizalde en la ciudad de Guayaquil, Ecuador.

METODOLOGÍA

Se realizó un estudio de prevalencia de niños con diagnóstico de testículo no descendido no palpable en el hospital de niños Dr. Roberto GilbertE. parte del complejo hospitalario Alejandro Mann en la ciudad de Guayaquil, Ecuador, durante el periodo 01 de julio de 2014 al 31 de marzo de 2018.
Se identificaron 639 pacientes con testículos no descendidos y 37 de este grupo con testículo no palpable, 4 con afectación bilateral y 33 con testículos unilaterales impalpables que fueron manejados por laparoscopía.

El protocolo utilizado en esta revisión consistió en examen clínico realizado por un urólogo pediátrico. Si al examen físico se diagnostica testículo no descendido no palpable, se solicita ecografía ínguino-pélvica. Si se mantiene el diagnóstico, se realiza una laparoscopía exploratoria.

La exploración laparoscópica se realizó mediante la inserción de un puerto de $5 \mathrm{~mm}$ infraumbilical utilizando técnicas cerradas, a través del cual se forma el neumoperitoneo ( 8 a $10 \mathrm{~mm} \mathrm{Hg}$ ) y se introduce una óptica de $30^{\circ}$. Se colocaron puertos secundarios bajo visión directa de 3,5 a $5 \mathrm{~mm}$ (dependiendo de la edad) que se sitúan en flanco derecho e izquierdo perpendiculares al primer puerto. Se intentó identificar los testículos, vasos testiculares, conducto y vasos deferentes. Además, si los anillos inguinales internos (IIRs) estaban abiertos o cerrados en busca de una hernia inguinal asociada.

Se clasificaron los hallazgos laparoscópicos de acuerdo con los patrones de estas estructuras y utilizados para determinar la gestión posterior. Una posición "baja" o canalicular se definió como siendo por debajo de los vasos iliacos externos; la orquidopexia sin sección de los vasos espermáticos, que consiste en la liberación del gubernaculum testis e incisión del peritoneo parietal posterior para movilizar los vasos espermáticos y el conducto deferente; cuando se conseguía una longitud que permitía llevar el testículo al orificio inguinal interno contralateral descendemos el teste al escroto a través del orificio inguinal interno.

Una posición "alta" de los testículos se definió como siendo por encima de los vasos iliacos externos; la orquidopexia para estos pacientes, consistió en un procedimiento de Fowler-Stephens en una etapa. Esta técnica consiste ocluir los vasos espermáticos con endoclips a tres centímetros del teste.

La orquiectomía se realizó ante un testículo evanescente o atrófico acompañado de un testículo contralateral normal. Todos los pacientes fueron seguidos a las 6 semanas, a los 6 meses y hasta los 2 años posteriores al procedimiento. 
Se documentaron detalles demográficos, lado del testículo impalpable, localización del testículo, hallazgos clínicos y laparoscópicos, hallazgos anatomopatológicos, intervención operativa y resultados.

Finalmente los datos fueron procesados en IBM SPSS Statistics versión 25. Para los aspectos éticos se obtuvo la aprobación para el estudio por parte del departamento de docencia del hospital y dado que se revisaron historias clínicas de manera retrospectiva, hubo exención de consentimiento informado para el estudio, y no existe conflicto de intereses por partes de los autores.

RESULTADOS

En el hospital de niños Dr. Roberto Gilbert Elizalde se reportaron desde el 01 de julio de 2014 al 31 de marzo de 2018, un total de 639 pacientes con testes no descendidos, de los cuales 37 eran no palpables $(6 \%)$. De este porcentaje de testículos no palpables, 4(11\%) con afectación bilateral y $33(89 \%)$ con testículos unilaterales. De los testículos unilaterales afectos, en $17(46 \%)$ ocasiones fue el derecho y en $16(43 \%)$ ocasiones el izquierdo. La distribución del grupo etario en: < 1 año, 1 a 5 años, 6 a 10 años y >10 años; en los cuales se vieron afectados $3(8 \%), 18(49 \%), 11(30 \%)$ y $5(14 \%)$ pacientes respectivamente.

En cuanto a la localización del testículo durante la valoración laparoscópica se encontraron $15(37 \%)$ a nivel del anillo inguinal interno y 26(63\%) intraabdominales.

En la figura 1 se observa la relación entre la edad del paciente y la localización del testículo no palpable. En el grupo de localización intraabdominal se distribuye: < 1 año, 2 casos (7\%); 1 a 5 años, 14 casos (37\%); 6 a 10 años, 5 casos (12\%) y $>10$ años, 2 casos $(7 \%)$. En el grupo de localización a nivel del anillo inguinal interno se reparten en: $<1$ año, 1 caso (5\%); 1 a 5 años, 6 casos (15\%); 6 a 10 años, 7 casos $(17 \%)$ y $>10$ años 0 casos.

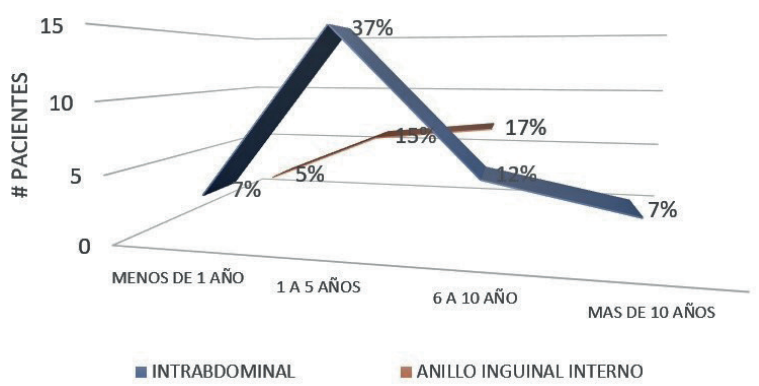

Figura 1. Relación entre la edad y la localización del testículo no palpable
La intervención operativa en base a los criterios previamente mencionados fueron $20(49 \%)$ orquidopexia sin sección de los vasos espermáticos, $11(27 \%)$ Fowler-Stephen y 10(24\%) orquiectomías. Por último, durante el análisis de la anatomía patológica se encontraron $7(17 \%)$ testículos evanescentes, $3(7 \%)$ atróficos, 5(12\%) hipotróficos, 22(54\%) hipoplásicos y 4(10\%) normales.

En la figura 2 se observa la relación entre la edad del paciente y la anatomía patológica. En el caso del testículo evanescente < 1 año, 2 casos (14\%); 1 a 5 años, 4 casos (28\%); 6 a 10 años, 1 caso (7\%) $\mathrm{y}>10$ años, 0 casos ( $0 \%$ ). Testículo hipotrófico $<1$ año, 0 casos ( $0 \%$ ); 1 a 5 años, 2 casos (14\%); 6 a 10 años, 2 caso ( $14 \%$ y y $>10$ años, 1 caso ( $7 \%$. Testículo atrófico < 1 año, 0 casos ( $0 \%$; 1 a 5 años, 0 casos $(0 \%) ; 6$ a 10 años, 2 casos (14\%) y >10 años, 1 caso $(7 \%)$.

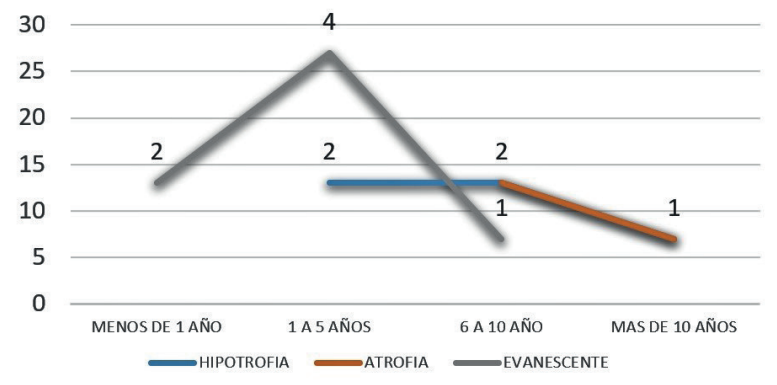

Figura 2. Relación entre la edad y la anatomía patológica del testículo no palpable

Por último, 2 (18\%) de los 11 pacientes operados con técnica de Fowler-Stephen presentaron atrofia testicular y se les realizó orquiectomía transescrotal.

\section{DISCUSIÓN}

El descenso testicular, aunque aún no se comprende del todo, toma lugar durante dos etapas gestacionales diferentes, ocurriendo durante las semanas intrauterinas 8 a 15 y de la 25 a 35. El fracaso de la primera fase del descenso es más rara que la segunda fase y da como resultado un testículo intraabdominal no descendido. ${ }^{17} \mathrm{El}$ criptorquidismo es el trastorno genitourinario más común del varón. A pesar de ésto, el manejo de niños con testículos palpables ha sido estandarizado, pero, no existen pautas formales para el manejo de los niños con testículos no palpables. ${ }^{18}$ Desde que Jordán introdujo la aplicación de la laparoscopía para el tratamiento de los testes intraabdominales, la comunidad urológica pediátrica la ha adoptado casi de forma universal, aunque actualmente aún hay autores que no la 
efectúan como Daher y cols. que realiza todos los descensos de forma descrita vía inguinal, desestimando incluso la sección de los vasos espermáticos en aquellos que quedan a tensión. ${ }^{19,20}$

La laparoscopía es actualmente el método diagnóstico más confiable en el manejo de los testículos no palpables ya que claramente muestra la anatomía y proporciona información visual sobre la que pueden basarse una decisión definitiva. ${ }^{21}$ Los dos principales hallazgos laparoscópicos posibles son: testículo intraabdominal, observado en el 63\% de los pacientes; a nivel de las estructuras del anillo inguinal interno, observado en $37 \% .{ }^{22}$ Aunque el lado derecho es más frecuente en los testículos no descendidos (45\%) en comparación con el lado izquierdo (35\%), Hemos encontrado en el presente estudio que el $43 \%$ de los pacientes con los testículos unilaterales no palpables estaban en el lado izquierdo, mientras que el $46 \%$ en el lado derecho, sin demostrarse una diferencia estadísticamente significativa.

En la actualidad, no hay consenso sobre cuál de estos procedimientos es el más beneficioso para corregir el TIAb, no obstante la técnica que se realiza idealmente, es a través de un abordaje laparoscópico o abierto. ${ }^{23}$

El presente estudio reveló testículos normales en el 10\% de los casos, testículos hipoplásicos en el $54 \%$ de los casos y entre hipotróficos y atróficos un total de 19\%. La morfología (anatomía patológica en los casos que ameritaban) se correlacionó con la edad del paciente, donde se reveló que las características de hipotrofia y atrofia eran altas en los testículos intraabdominales a mayor edad del paciente.

No se observaron complicaciones quirúrgicas mayores en este estudio; se evidenció de atrofia testicular en el $18 \%$ de los pacientes operados con técnica de Fowler-Stephen con seguimiento durante el tiempo de estudio.

\section{CONCLUSIONES Y RECOMENDACIONES}

En conclusión, la laparoscopía es una herramienta extremadamente útil y modalidad segura tanto para el diagnóstico como para el manejo de testículos no descendidos no palpables. Además de ser un excelente indicador intraoperatorio para decidir sobre el tipo de orquidopexia, en base a la localización del testículo y las características morfológicas que presente el mismo. Y en todos los casos, es necesario un seguimiento a largo plazo para controlar el estado del testículo después de la cirugía. También es necesario mencionar que la edad de diagnóstico es tardía, motivo por el cual se encuentran una gran cantidad de testículos atróficos, hipotróficos e hipoplásicos. Y es importante que se lo haga en edades menores del promedio de este estudio.

\section{REFERENCIAS BIBLIOGRÁFICAS}

1. Cortes D, Kjellberg EM, Breddam M, Thorup J (2008) The true incidence of cryptorchidism in Denmark. JUrol 179(1):314-318

2. Thonneau PF, Gandia P, Mieusset R. Cryptorchidism: incidence, risk factors, and potential role of environment; an update an update. J Androl. Mar-Apr 2003;24(2):155-62

3. Thong M, Lim C, Fatimah H (1998) Undescended testes: incidence in 1,002 consecutive male infants and outcome at 1 year of age. Pediatr Surg Int 13(1):37-41

4. Smolko MJ, Kaplan GW, Brock WA (1983) Location and fate of the nonpalpable testis in children. JUrol 129(6):1204-1206

5. Comploj E, Pycha A (2012) Diagnosis and management of cryptorchidism. Eur Urol Suppl 11(2):2-9

6. Batata M, Whitmore W Jr, Hilaris B, Tokita N, Grabstald H (1976) Cancer of the undescended or maldescended testis. Am J Roentgenol 126(2):302-312

7. AbouZeid AA, Mousa MH, Soliman HA, Hamza AF, Hay SA (2011) Intra-abdominal testis: histological alterations and significance of biopsy. J Urol 185(1):269-274

8. Hvistendahl GM, Poulsen EU (2009) Laparoscopy for the impalpable testes: experience with $80 \mathrm{in}^{-}$ tra-abdominal testes. J Pediatr Urol 5(5):389-392

9. Murphy F, Paran TS, Puri P (2007) Orchidopexy and its impact on fertility. Pediatr Surg Int 23(7):625-632

10. Carson JS, Cusick R, Mercer A, Ashley A, Abdessalam S, Raynor S, Lyden E, Azarow K (2014) Undescended testes: does age at orchiopexy affect survival of the testis? J Pediatr Surg 49(5):770-773

11. Canavese F, Mussa A, Manenti M, Cortese MG, Ferrero L, Tuli G, Macchieraldo R, Lala R (2009) Sperm count of young men surgically treated for cryptorchidism in the first and second year of life: fertility is better in children treated at a younger age. Eur J Pediatr Surg 19(6):388-391

12. Pettersson A, Richiardi L, Nordenskjold A, Kaijser M, Akre O (2007) Age at surgery for undescended testis and risk of testicular cancer. $\mathrm{N}$ Engl J Med 356(18):1835-1841 
13. Fowler R, Stephens FD (1959) The role of testicular vascular anatomy in the salvage of high undescended testes. Aust N Z J Surg 29:92-106

14. Ransley PG, Wacksman JS, Caldamone AA, Bellinger MF (1984) Preliminary ligation of the gonadal vessels prior to orchidopexy for the intra-abdominal testicle. World JUrol 2:266-268

15. Cortesi N, Baldini A, Ferrari P, et al. Diagnosis of bilateral abdominal cryptorchidism by laparoscopy. Endoscopy. 1976; 8:334.

16. Siemers S, Humke U, et al. Diagnosis of nonpalpable testes in childhood: comparison of magnetic resonance imaging and laparoscopy in a perspective study. Eur J Pediatre Surg. 2000;10 (2): 114-8.

17. Deshpande AV, Michail P, Smith GH. Interventions for undescended testes in children. Cochrane Database of Systematic Reviews 2014, Issue 2. Art. No.: CD010978. DOI: 10.1002/14651858.CD010978.

18. Hutson J. M. and Clarke M. C. "Current management of the undescended testicle," Seminars in Pediatric Surgery, vol. 16, no. 1, pp. 64-70, 2007.

19. Daher P, Nabbout P, Feghali J, Riachy E. Is the Fowler-Stephens procedure still indicated for the treatment of nonpalpable intraabdominal testis? J Pediatr Surg. 2009 Oct;44(10):1999-2003.

20. Jordan JH, Robey EL, Winslow BH. Laparoendoscopic surgical management of the abdominal/ transinguinal undescended testicle. JEndourol. 1992; 6: 157-61.

21. Esposito C, Caldamone A, Settimi A, and El-Choneimi A, "Management of boys with nonpalpable undescended testis," Nature Clinical Practice Urology, vol. 5, no. 5, pp. 252-260, 2008.

22. Tennenbaum S. Y., Lerner S. E., McAleer I. M., Packer M. G, Scherz H. C., and Kaplan G. W., "Preoperative laparoscopic localization of the nonpalpable testis: a critical analysis of a 10year experience," Journal of Urology, vol. 151, no. 3, pp. 732-734, 1994.

23. Hassan M. E. and Mustafawi A, "Laparoscopic management of impalpable testis in children, new classifica-tion, lessons learned, and rare anomalies," Journal of Laparoendoscopic \& Advanced Surgical Techniques A, vol. 20, no. 3, pp. 265-269, 2010. 